\title{
Progression of ascending aortopathy may not occur after transcatheter aortic valve replacement in severe bicuspid aortic stenosis
}

\author{
Ji-Hyun Jung, Hyung-Kwan Kim, Jun-Bean Park, Seung-Pyo Lee, Bon-Kwon Koo, Yong-Jin Kim, \\ Hyo-Soo Kim, and Dae-Won Sohn
}

Division of Cardiology, Cardiovascular Center, Department of Internal Medicine, Seoul National University Hospital, Seoul, Korea

Received: March 15, 2019

Revised : May 19, 2019

Accepted: June 16, 2019

\section{Correspondence to}

Hyung-Kwan Kim, M.D.

Division of Cardiology, Cardiovascular Center, Department of Internal Medicine, Seoul National University College of Medicine, 101 Daehak-ro, Jongno-gu, Seoul 03080, Korea

Tel: +82-2-2072-0243

Fax: $+82-2-766-8904$

E-mail: hkkim73@snu.ac.kr

https://orcid.org/0000-0001-

7950-2131
Background/Aims: We evaluated changes in the ascending aorta dimension post-transcatheter aortic valve replacement (TAVR) in bicuspid aortic valve (BiAV) and tricuspid aortic valve (TAV) patients.

Methods: Patients with severe aortic stenosis undergoing TAVR at Seoul National University Hospital were consecutively recruited. Patients with less than 12 months' follow-up and/or with an ascending aorta size larger than $50 \mathrm{~mm}$ were excluded. The ascending aorta size was measured on a parasternal long axis view using transthoracic echocardiography.

Results: Among the 67 patients who were included (age: $76.5 \pm 6.5$ years; male: 52.2\%; AV area: $\left.0.67 \pm 0.15 \mathrm{~cm}^{2}\right), 19$ (28.4\%) had BiAV; 48 (71.6\%) had TAV. The median (interquartile ranges) follow-up duration was 398 days (361 to 451 ). BiAV patients were younger $(73.2 \pm 7.2 \mathrm{vs} .77 .8 \pm 5.8, p=0.008)$, and had lower incidences of chronic renal disease (5.3\% vs. $35.4 \%, p=0.014$ ) and history of coronary intervention ( $15.8 \%$ vs. $50.0 \%, p=0.013)$, than TAV patients. On pre-procedural echocardiography, the ascending aorta dimensions in BiAV patients were larger than those in TAV patients $(40.5 \pm 3.8 \mathrm{~mm}$ vs. $35.9 \pm 4.2 \mathrm{~mm}, p<0.005)$. The ascending aorta dimension changed minimally during follow-up; post-TAVR, the ascending aorta's growth rate was $-0.11 \pm 1.9$ and $0.26 \pm 1.8 \mathrm{~mm} / \mathrm{yr}$ in patients with BiAV and TAV, respectively $(p=0.50)$. Progression of the ascending aorta's dimension postTAVR was not clinically significant in BiAV patients.

Conclusions: The concern about the progression of aortopathy in BiAV patients post-TAVR may not be a clinical issue. This should be confirmed in studies with a larger population and with a longer follow-up duration.

Keywords: Aortic valve stenosis; Transcatheter aortic valve replacement; Bicuspid; Aortic aneurysm

\section{INTRODUCTION}

Transcatheter aortic valve replacement (TAVR) is now widely performed not only in patients at high risk, but also in those at intermediate risk who have severe aor- tic valve stenosis (AS) of the tricuspid aortic valve (TAV) $[1,2]$. Since altered valve morphology in the bicuspid aortic valve (BiAV) makes it more susceptible to mechanical stress, AS is a common complication in patients with $\mathrm{BiAV}$. In elderly AS population, the prevalence of BiAV 
is reported to be relatively high, and $22 \%$ of octogenarians and $18 \%$ of nonagenarians have experienced surgical aortic valve (AV) replacement for treatment option [3]. Thus, this specific subset comprises significant proportion of people with AS. Although most of the major randomized clinical trials have excluded patients with AS of the BiAV, a few observational studies support the application of TAVR in AS related to the BiAV [4-6].

The BiAV is commonly associated with ascending aortic dilation, which can progress to aortic aneurysm and dissection [7-11]. Ascending aortic pathology may progress even after a successful TAVR procedure, which is one of the reasons why AS patients with a BiAV have been excluded in previous clinical trials. No data, however, are available on changes in the ascending aorta after TAVR in patients with severe AS of the BiAV. The aim of this study, therefore, was to evaluate changes occurring in the ascending aorta following TAVR in AS patients with a BiAV compared with those with a TAV. Demonstration of the absence of ascending aortopathy progression in patients with a BiAV makes clinicians feel comfortable, and TAVR may be actively recommended without concern of ascending aortopathy progression in this particular subset.

\section{METHODS}

\section{Study population}

Patients with severe AS who underwent TAVR between July 2011 and December 2016 at Seoul National University Hospital were consecutively enrolled. Patients with clinical and echocardiographic follow-up periods less than 12 months (window period for 12-month follow-up $= \pm 2$ weeks) and/or with an ascending aorta size larger than $50 \mathrm{~mm}$ were excluded. We systematically obtained data on clinical characteristics including sex, height, weight, body surface area, medical history (stroke, myocardial infarction, peripheral artery disease, and chronic renal disease), and laboratory data. Chronic renal disease was defined as an estimated glomerular filtration rate less than $60 \mathrm{~mL} / \mathrm{min}$, derived from Modification of Diet in Renal Disease equation. The Society of Thoracic Surgeons score and EuroSCORE II were also calculated. The study was performed in accordance with the ethical principles of the Declaration of Helsinki and registered at clinicaltrials.gov (study identifier: NCTo3051334). The Institutional Review Board of Seoul National University Hospital (1701-104-825) approved the study protocol, and informed consent was obtained.

\section{Imaging modalities}

Pre-procedural transthoracic echocardiography (TTE), transesophageal echocardiography (TEE), and computed tomography (CT) data were obtained. The left ventricu$\operatorname{lar}(\mathrm{LV})$ ejection fraction, LV dimensions, wall thickness, valvular dysfunction, and ascending aorta dimension were evaluated using TTE. Hemodynamic data including systolic and diastolic blood pressure, and heart rate during TTE were also collected. Valvular dysfunction was classified as mild, moderate, or severe. Severe AS was diagnosed according to the American Society of Echocardiography guideline [12]. BiAV was diagnosed when only two cusps or definite raphe was identified on both echocardiography and CT. The ascending aorta's dimension was measured on TTE around $3 \mathrm{~cm}$ distal from the sinotubular junction in a parasternal long axis view by using the inner edge-to-inner edge convention.

An electrocardiogram-gated CT imaging was obtained before TAVR. Images were reconstructed during the end-diastolic and mid-systolic phases. We measured the aortic root, annulus, and mid-ascending aorta using a reconstructed image of the mid-systolic phase.

\section{TAVR}

TAVR was performed with the patient under general anesthesia, using TEE guidance. Transfemoral or transapical access was selected according to the valve size, vascular calcification, tortuosity of the aorta, and arteriosclerosis obliterans of the iliofemoral artery. An Edwards SAPIEN valve, CoreValve, or Lotus valve was used. The valve size was selected according to the dimensions of the annulus and aortic root, and measured with CT and echocardiography. The Edwards valve had a diameter of 23 to $29 \mathrm{~mm}$, the CoreValve's diameter was 23 to $31 \mathrm{~mm}$, and the Lotus valve's diameter was 23 to $29 \mathrm{~mm}$.

Device success was defined according to the Consensus Report from the Valve Academic Research Consortium [13], as follows: successful vascular access; successful delivery and deployment of the device; correct position of the device in the proper anatomical location; intended performance of the prosthetic valve (e.g., AV area >1.2 
$\mathrm{cm}^{2}$; mean AV gradient < $20 \mathrm{mmHg}$; or AV peak velocity $<3 \mathrm{~m} / \mathrm{sec}$ ), without moderate or severe paravalvular leakage; and only one valve that was implanted in the proper anatomical location. Procedure-related complications, including death, acute stroke, new pacemaker implantation, vascular complication, aortic root injury, and coronary obstruction, were reported. A major vascular complication was defined as any aortic dissection, access site or access-related vascular injury leading to death, a need for blood transfusions ( $\geq 4$ units), or an additional, unplanned surgical intervention.

\section{Follow-up and outcomes}

The follow-up duration is expressed as the median (interquartile ranges [IQR]). The annual growth rate of the ascending aorta $(\mathrm{mm} / \mathrm{yr})$ was calculated as the changes in the aortic dimension divided by the follow-up period. TTE and TEE were performed at pre-specified time-points ( 6 and 12 months post-TAVR) to evaluate the function of the prosthetic valve. The mean pressure gradient of the prosthetic $\mathrm{AV}$, the presence of $\mathrm{AV}$ regurgitation, LV ejection fraction, and the dimension of the ascending aorta were evaluated. Paravalvular regurgitation was qualitatively evaluated based on TTE and TEE, and classified as none, mild, moderate, or severe [14].

\section{Statistical analyses}

Continuous variables are expressed as the mean \pm standard deviation or median value with IQR, and categorical variables are expressed as percentages, when appropriate. An independent $t$ test was used to compare continuous variables, and the Mann-Whitney $U$ test was used to analyze non-normally distributed variables. A paired $t$ test was used to compare continuous variables between the baseline and follow-up echocardiographic variables after TAVR. Fisher's exact test was adopted to compare categorical variables. The difference in the ascending aorta growth rates between the two groups was analyzed using an independent $t$ test. The growth rates between patients with and without ascending aortic aneurysm was analyzed using an independent $t$ test. All statistical analyses were performed using SPSS statistics version 22.0 (IBM Co., Armonk, NY, USA) and a p value < 0.05 was considered statistically significant.

\section{RESULTS}

\section{Patient characteristics}

Seventy-nine patients were initially included, 12 of whom were excluded owing to a short follow-up dura-

Table 1. Clinical characteristics of study population

\begin{tabular}{|c|c|c|c|c|}
\hline Clinical parameter & $\operatorname{BiAV}(\mathrm{n}=19)$ & $\operatorname{TAV}(\mathrm{n}=48)$ & Total $(\mathrm{n}=67)$ & $p$ value \\
\hline Age, yr & $73.2 \pm 7.2^{\mathrm{a}}$ & $77.8 \pm 5.8^{\mathrm{a}}$ & $76.5 \pm 6.5$ & 0.008 \\
\hline Male sex & $12(63.2)$ & $23(47.9)$ & $35(52.2)$ & 0.29 \\
\hline $\mathrm{BMI}, \mathrm{kg} / \mathrm{m}^{2}$ & $23.9 \pm 2.7$ & $23.9 \pm 3.4$ & $23.9 \pm 3.2$ & 0.96 \\
\hline Hypertension & $12(63.2)$ & $39(81.3)$ & $51(76.1)$ & 0.20 \\
\hline Diabetes & $5(26.3)$ & $25(31.3)$ & $20(29.9)$ & 0.77 \\
\hline Previous stroke & $2(10.5)$ & $9(18.8)$ & $11(16.4)$ & 0.72 \\
\hline Previous MI & 0 & $2(4 \cdot 2)$ & $2(3.0)$ & 1.00 \\
\hline Previous PCI & $3(15.8)^{\mathrm{a}}$ & $24(50.0)^{\mathrm{a}}$ & $27(40.3)$ & 0.013 \\
\hline Peripheral artery disease & $1(5 \cdot 3)$ & $2(4.2)$ & $3(4 \cdot 5)$ & 1.00 \\
\hline Chronic kidney disease & $1(5 \cdot 3)^{\mathrm{a}}$ & $17(35 \cdot 4)^{\mathrm{a}}$ & $18(26.9)$ & 0.014 \\
\hline STS score & $2.6(1.8-7.4)$ & $3.8(2.3-11.9)$ & $3.5(2.2-9.6)$ & 0.13 \\
\hline EuroSCORE II & $1.1(0.9-2.3)^{\mathrm{a}}$ & $1.8(1.4-2.5)^{\mathrm{a}}$ & $1.6(1.2-2.6)$ & 0.02 \\
\hline
\end{tabular}

Values are presented as mean $\pm \mathrm{SD}$, number (\%), or median (interquartile ranges). Student's $t$ test, Fisher's exact test, or Mann-Whitney $U$ test were used for comparison between groups.

BiAV, bicuspid aortic valve; TAV, tricuspid aortic valve; BMI, body mass index; MI, myocardial infarction; PCI, percutaneous coronary intervention; STS, The Society of Thoracic Surgeons.

a $p<0.05$ between BiAV and TAV. 
tion and/or lack of appropriate echocardiographic images. Among the 67 patients who underwent TAVR (age: $76.5 \pm 6.5$ years; male: $52.2 \%$; AV area: $\left.0.67 \pm 0.15 \mathrm{~cm}^{2}\right), 19$ (28.4\%) had BiAV; 48 (71.6\%) had TAV.

The baseline characteristics of these participants are summarized in Table 1. Patients with a BiAV were younger than patients with a TAV $(73.2 \pm 7.2$ years vs. $77.8 \pm 5.8$ years, $p=0.008$ ), and had a lower incidence of chronic kidney disease (5.3\% vs. $35.4 \%, p=0.014$ ) and history of previous coronary intervention ( $15.8 \%$ vs. $50.0 \%$, $p=0.013)$. The baseline echocardiographic variables are illustrated in Table 2. As expected, the dimensions of sinus of Valsalva and ascending aorta were larger in patients with a BiAV than in those with a TAV. The preva- lence of ascending aortic dilation $(\geq 40 \mathrm{~mm}$ ) was higher in patients with a BiAV than in those with a TAV $(57.9 \%$ vs. $16.7 \%, p=0.002$ ).

\section{Procedural characteristics and complications}

The procedural characteristics are listed in Table 3. The device success rate was similar for both groups (100\% vs. 89.6\% for patients with a BiAV and TAV, respectively; $p=$ 0.31). Three patients with a TAV required a second valve deployment. Immediately after TAVR, two patients with a TAV and severe AS had a paravalvular regurgitation grade that was greater than mild. A CoreValve was used for both patients. A newly developed conduction system problem necessitating implantation of a permanent

Table 2. Echocardiographic parameters before and after transcatheter aortic valve replacement

\begin{tabular}{|c|c|c|c|c|}
\hline Variable & $\operatorname{BiAV}(n=19)$ & $\mathrm{TAV}(\mathrm{n}=48)$ & $\operatorname{Total}(\mathrm{n}=67)$ & $p$ value \\
\hline \multicolumn{5}{|l|}{ Pre-TAVR } \\
\hline $\mathrm{AV}$ area, $\mathrm{cm}^{2}$ & $0.68 \pm 0.17$ & $0.67 \pm 0.15$ & $0.67 \pm 0.15$ & 0.77 \\
\hline Mean AV pressure gradient, $\mathrm{mmHg}$ & $71.0 \pm 15.0^{\mathrm{a}}$ & $55.8 \pm 15 \cdot 3^{\mathrm{a}}$ & $58.6 \pm 16.3$ & 0.008 \\
\hline LV ejection fraction, $\%$ & $60.4 \pm 4.7$ & $58.5 \pm 7.6$ & $59.1 \pm 6.9$ & 0.34 \\
\hline Ascending aorta size (echo-measured), mm & $40.5 \pm 3.8^{\mathrm{a}}$ & $35 \cdot 9 \pm 4.2^{\mathrm{a}}$ & $37 \cdot 3 \pm 4.6$ & $<0.005$ \\
\hline Ascending aorta size (CT-measured), mm & $41.7 \pm 3.9^{\mathrm{a}}$ & $37.1 \pm 4.0^{\mathrm{a}}$ & $38.4 \pm 4.5$ & $<0.005$ \\
\hline Aortic annulus, $\mathrm{mm}$ & $21.7 \pm 1.5$ & $21.0 \pm 1.6$ & $21.2 \pm 1.6$ & 0.057 \\
\hline Sinus of Valsalva, $\mathrm{mm}$ & $34 \cdot 9 \pm 3.6^{\mathrm{a}}$ & $32.1 \pm 3.9^{\mathrm{a}}$ & $32.9 \pm 4.1$ & 0.009 \\
\hline AR more than mild & $1(5 \cdot 3)$ & $5(10.4)$ & $6(9.0)$ & 0.67 \\
\hline MR more than mild & 0 & $1(2.1)$ & $1(1.5)$ & 1.0 \\
\hline \multicolumn{5}{|l|}{ Six months after TAVR } \\
\hline Prosthetic AV area, $\mathrm{cm}^{2}$ & $1.8 \pm 0.5$ & $1.7 \pm 0.4$ & $1.7 \pm 0.4$ & 0.21 \\
\hline Mean AV pressure gradient, $\mathrm{mmHg}$ & $13 \cdot 4 \pm 3 \cdot 7$ & $11.0 \pm 4.4$ & $11.4 \pm 4.4$ & 0.08 \\
\hline LV ejection fraction, \% & $62.3 \pm 4.4$ & $60.5 \pm 4.2$ & $61.0 \pm 4.3$ & 0.13 \\
\hline Ascending aorta size (echo-measured), mm & $40.7 \pm 4 \cdot 7^{\mathrm{a}}$ & $36.9 \pm 4.0^{\mathrm{a}}$ & $38.0 \pm 4.5$ & $<0.005$ \\
\hline \multicolumn{5}{|l|}{ Twelve months after TAVR } \\
\hline Prosthetic AV area, $\mathrm{cm}^{2}$ & $1.8 \pm 0.6$ & $1.7 \pm 0.4$ & $1.8 \pm 0.5$ & 0.56 \\
\hline Mean AV pressure gradient, $\mathrm{mmHg}$ & $13.1 \pm 5.6$ & $10.5 \pm 4.0$ & $11.2 \pm 4.6$ & 0.050 \\
\hline LV ejection fraction, \% & $62.9 \pm 4.7$ & $62.5 \pm 3.9$ & $62.7 \pm 4.1$ & 0.75 \\
\hline Ascending aorta size (echo-measured), mm & $40.6 \pm 3.7^{\mathrm{a}}$ & $35.9 \pm 3.9^{\mathrm{a}}$ & $37.2 \pm 5.7$ & $<0.005$ \\
\hline Changes in ascending aorta, $\mathrm{mm} / \mathrm{yr}$ & $-0.11 \pm 1.9$ & $0.26 \pm 1.8$ & $0.16 \pm 1.8$ & 0.50 \\
\hline Changes in ascending aorta by CT $(\mathrm{n}=30), \mathrm{mm} / \mathrm{yr}$ & $-0.19 \pm 0.5$ & $0.14 \pm 0.4$ & $0.02 \pm 0.5$ & 0.051 \\
\hline
\end{tabular}

Values are presented as mean \pm SD or number (\%). Student's $t$ test or Fisher's exact tests were used for comparison between groups.

BiAV, bicuspid aortic valve; TAV, tricuspid aortic valve; TAVR, transcatheter aortic valve replacement; AV, aortic valve; LV, left ventricle; CT, computed tomography; AR, aortic regurgitation; MR, mitral regurgitation.

${ }^{\mathrm{a}} \mathrm{p}<0.05$ between BiAV and TAV. 
pacemaker occurred in two patients (3.3\%) with a TAV (both of them had CoreValve). One patient with TAV-related AS who experienced major vascular complications needed surgical repair owing to inadequate vascular closure after TAVR. In addition, we incidentally observed descending thoracic aortic dissection on a follow-up CT angiography scan in one patient with a BiAV. Neither coronary obstruction nor aortic root injury occurred in patients enrolled in this study.

\section{Follow-up and durability}

At 12 months' follow-up, the effective orifice area of the implanted prosthetic AV was $1.8 \pm 0.5 \mathrm{~cm}^{2}$, the mean pressure gradient was $11.2 \pm 4.6 \mathrm{mmHg}$, and the maximal

Table 3. Procedural characteristics of study population

\begin{tabular}{|c|c|c|c|c|}
\hline Characteristic & $\operatorname{BiAV}(\mathrm{n}=19)$ & $\operatorname{TAV}(\mathrm{n}=48)$ & Total $(\mathrm{n}=67)$ & $p$ value \\
\hline \multicolumn{5}{|l|}{ Procedural characteristic } \\
\hline Valve type & & & & 0.71 \\
\hline Edwards & $3(15.8)$ & $12(25.0)$ & $15(22.4)$ & \\
\hline CoreValve & $14(73.7)$ & $32(66.7)$ & $46(68.7)$ & \\
\hline Lotus & $2(10.5)$ & $4(8.3)$ & $6(9.0)$ & \\
\hline Access & & & & 0.31 \\
\hline Transfemoral & $19(100)$ & $43(89.6)$ & $62(92.5)$ & \\
\hline Transapical & o & $5(10.4)$ & $5(7 \cdot 5)$ & \\
\hline \multicolumn{5}{|l|}{ Valve size } \\
\hline Edwards & & & & 0.39 \\
\hline 23 & $\mathrm{O}$ & $3(25.0)$ & $3(20.0)$ & \\
\hline 26 & $2(66.7)$ & $8(66.7)$ & $10(66.7)$ & \\
\hline 29 & $1(33 \cdot 3)$ & $1(8.3)$ & $2(13 \cdot 3)$ & \\
\hline CoreValve & & & & $<0.005$ \\
\hline 23 & $3(21.4)^{\mathrm{a}}$ & $1(3.1)^{\mathrm{a}}$ & $4(8.7)$ & \\
\hline 26 & $1(7 \cdot 1)^{\mathrm{a}}$ & $22(68.8)^{\mathrm{a}}$ & $23(50.0)$ & \\
\hline$\geq 29$ & $10(71.4)^{\mathrm{a}}$ & $9(28.2)^{\mathrm{a}}$ & $19(41.3)$ & \\
\hline Lotus & & & & 0.22 \\
\hline 23 & $1(50.0)$ & $2(50.0)$ & $3(50.0)$ & \\
\hline 25 & o & $2(50.0)$ & $2(33 \cdot 3)$ & \\
\hline$\geq 27$ & $1(50.0)$ & o & $1(16.7)$ & \\
\hline \multicolumn{5}{|l|}{ Procedural outcomes } \\
\hline Device success & $19(100)$ & $43(89.6)$ & $62(92.5)$ & 0.31 \\
\hline Need 2nd TAVR & o & $3(6.3)$ & $3(4 \cdot 5)$ & 0.55 \\
\hline Conversion to SAVR & o & o & o & 1.0 \\
\hline Acute stroke & 0 & $2(4 \cdot 9)$ & $2(3 \cdot 3)$ & 1.0 \\
\hline Major vascular complication & 0 & $1(2.4)$ & $1(1.7)$ & 1.0 \\
\hline New pacemaker & o & $2(4 \cdot 9)$ & $2(3 \cdot 3)$ & 1.0 \\
\hline Aortic root injury & 0 & o & o & 1.0 \\
\hline Coronary obstruction & 0 & 0 & 0 & 1.0 \\
\hline
\end{tabular}

Values are presented as number (\%). Fisher's exact tests were used for comparison between groups.

BiAV, bicuspid aortic valve; TAV, tricuspid aortic valve; TAVR, transcatheter aortic valve replacement; SAVR, surgical aortic valve replacement.

${ }^{\mathrm{a}} \mathrm{p}<0.05$ between BiAV and TAV. 
velocity through the prosthetic AV was $2.3 \pm 0.4 \mathrm{~m} / \mathrm{sec}$. Significant paravalvular regurgitation greater than mild was found in one patient.

The ascending aorta's dimension remained stable throughout 1 year of follow-up after TAVR. In patients with a BiAV, the ascending aorta's dimension was 40.7 $\pm 4.7 \mathrm{~mm}$ at 6 months and $40.6 \pm 3.7 \mathrm{~mm}$ at 12 months after TAVR $(p<0.005$ and $p<0.005$ vs. the baseline value), whereas in those with a TAV, the corresponding values were $36.9 \pm 4.0,35.9 \pm 3.9 \mathrm{~mm}$, respectively $(p<0.005$ and $p<0.005$ vs. the baseline value) (Table 2, Fig. 1).

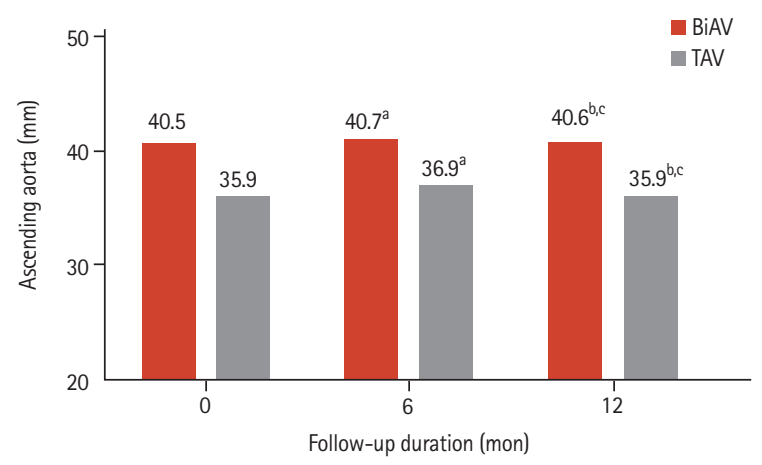

Figure 1. Changes in the ascending aorta's dimension after transcatheter aortic valve replacement. BiAV, bicuspid aortic valve; TAV, tricuspid aortic valve. ${ }^{\mathrm{a}} \mathrm{p}<0.05$ between baseline and 6 months after procedure. ${ }^{b} p<0.05$ between baseline and 12 months after procedure. ${ }^{c} p<0.05$ between 6 months and 12 months after procedure of ascending aorta's dimension.

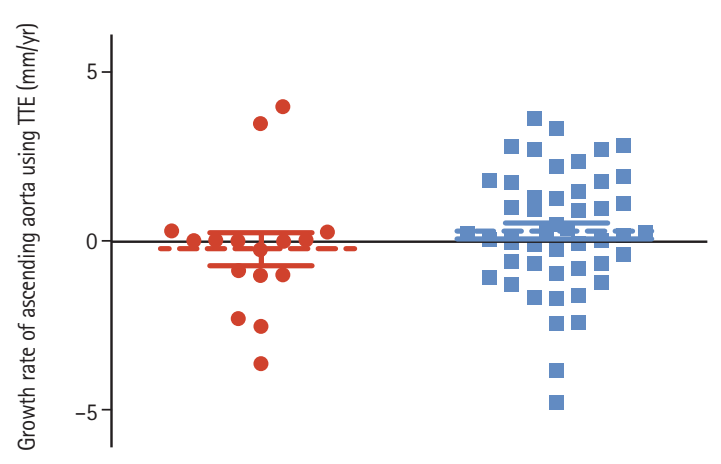

A BiAV

\section{The growth rate of the ascending aorta}

The median follow-up duration was 398 days (IQR, 361 to 451). The growth rate of the ascending aorta after TAVR was $-0.11 \pm 1.9 \mathrm{~mm} / \mathrm{yr}$ in patients with a BiAV and 0.26 $\pm 1.8 \mathrm{~mm} / \mathrm{yr}$ in those with a TAV $(p=0.50)$ (Fig. 2). The growth rates of the ascending aorta for both groups were minimal, and progression after TAVR, leading to the need for further treatment, was rare. Among them, CT aortography was performed in 11 patients with a BiAV and 19 with a TAV, for various reasons. The calculated growth rate of the ascending aorta using CT in BiAV patients showed a tendency to decrease compared with in TAV patients $(-0.07 \pm 0.48 \mathrm{~mm} / \mathrm{yr}$ vs. $0.14 \pm 0.38 \mathrm{~mm} / \mathrm{yr}$, respectively; $p=0.23$ ), but no statistical difference was observed. The measurement agreement between TTE and CT was excellent, as shown in Fig. $3 \mathrm{~A}(r=0.93, p<$ $0.001)$ and $3 \mathrm{~B}$.

The growth rate of the ascending aorta of all subjects was $0.16 \pm 1.8 \mathrm{~mm} / \mathrm{yr}$ (IQR, -0.90 to 1.27 ), which was not different from that reported in the normal population (IQR, 0.12 to $0.29 \mathrm{~mm} / \mathrm{yr}$ ) [15]. In the present study, 27 patients (40.9\%) (four patients with a BiAV [22.2\%] and 23 patients with a TAV [47.9\%]) had a growth rate of more than $0.3 \mathrm{~mm} / \mathrm{yr}$.

The growth rate of the ascending aorta is known to increase with a larger aortic dimension; the growth rate is reported to be approximately $1 \mathrm{~mm} / \mathrm{yr}$ in patients with an aortic aneurysm [16]. When we separated the patients according to the size of the ascending aorta, the growth

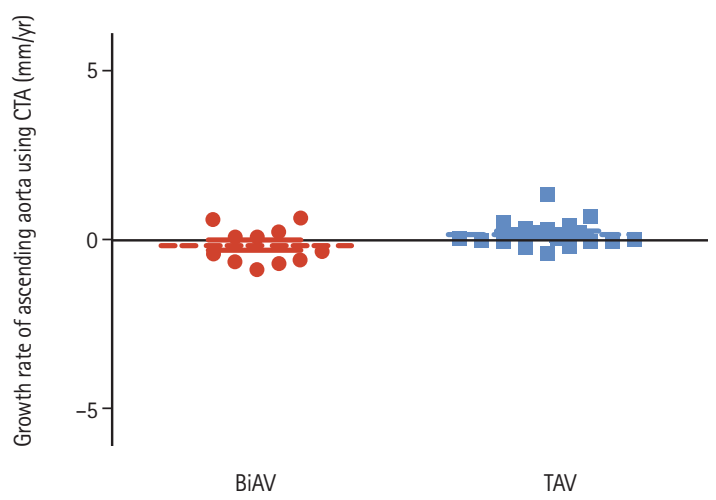

Figure 2. Growth rates of the ascending aorta's dimension after transcatheter aortic calve replacement, which were assessed using (A) transthoracic echocardiography (TTE) in all patients and (B) computed tomography angiography (CTA) in 30 patients. The red dots represent patients with a bicuspid aortic valve (BiAV), whereas the blue dots represent patients with a tricuspid aortic valve (TAV). The dashed lines represent the mean values of each group. The growth rates of the ascending aorta in patients with a BiAV showed that there were no significant differences compared with those in patients with a TAV. 

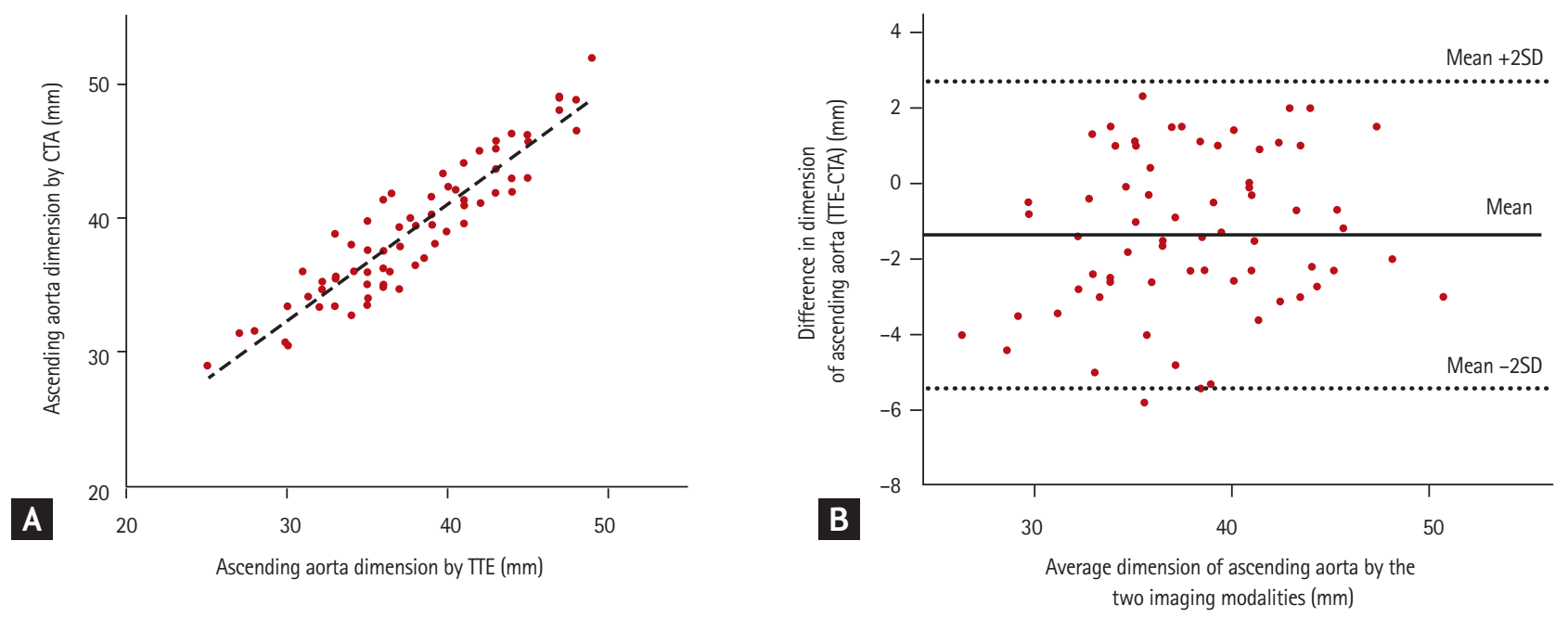

Figure 3. Comparison of the baseline ascending aorta dimension that was measured using transthoracic echocardiography (TTE) and computed tomography angiography (CTA). (A) The correlation between TTE and CTA in the measurement of the ascending aorta's dimension before transcatheter aortic valve replacement was good. (B) A Bland-Altman plot showed that there was excellent agreement between the two methods.

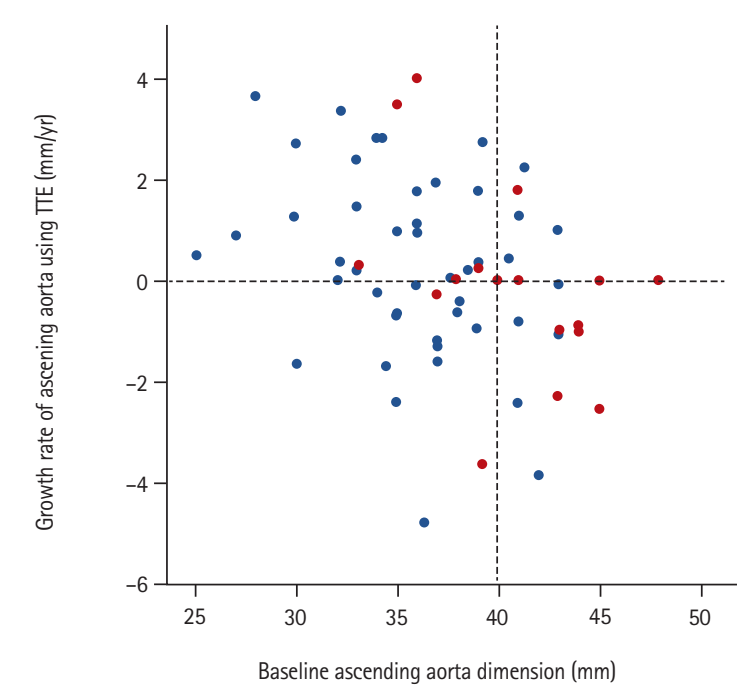

A

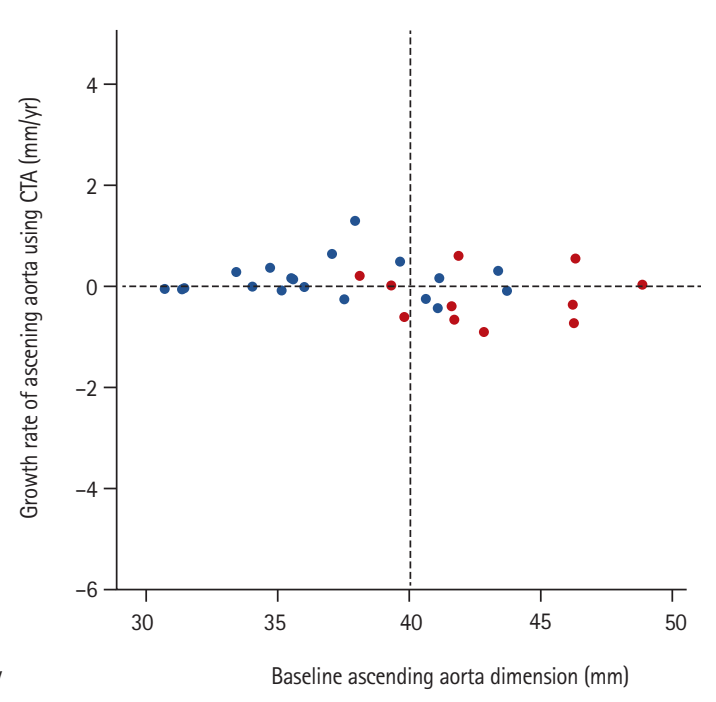

- BiAV - TAV

B

Figure 4. Growth rates of the ascending aorta's dimension according to the baseline ascending aorta size, assessed with (A) transthoracic echocardiography (TTE) and (B) computed tomography angiography (CTA). The red dots represent patients with a bicuspid aortic valve (BiAV), whereas the blue dots represent patients with a tricuspid aortic valve (TAV). The growth rate of the ascending aorta after the procedure cannot be predicted using the baseline ascending aorta dimension

rate of patients with an ascending aorta size $\geq 40 \mathrm{~mm}$ before the index TAVR procedure was smaller than that of the patients with an ascending aorta size $<40 \mathrm{~mm}$ $(-0.63 \pm 1.4 \mathrm{~mm} / \mathrm{yr}$ vs. $0.47 \pm 1.8 \mathrm{~mm} / \mathrm{yr}$, respectively; $p=$ ०.03) (Fig. 4). Moreover, progressive aortic dilation more than $1 \mathrm{~mm} / \mathrm{yr}$ was observed in only two AS patients (11\%) with an ascending aorta size $\geq 40 \mathrm{~mm}$.

\section{DISCUSSION}

To the best of our knowledge, this is the first study evaluating the stability of the ascending aorta's dimension after TAVR in patients with a BiAV and severe AS. The principal findings of the present study are summarized as follows:(1) the ascending aorta's dimension in patients 
with a BiAV and severe AS was significantly larger than that in patients with severe AS and a TAV before TAVR; and (2) the ascending aorta's dimension remained stable in patients with a BiAV and severe AS, without further dilation 12 months after TAVR. This finding suggests that concern about the progression of ascending aortopathy in patients with a BiAV should not be a reason for avoiding TAVR in patients with a BiAV and severe AS.

The safety and durability of TAVR are now clinically accepted, and thus its indication has expanded in patients with severe AS and a TAV. However, TAVR is at the center of debate in patients with severe AS and a $\mathrm{BiAV}$, especially between cardiologists and cardiac surgeons $[5,6,17-20]$.

BiAV was an exclusion of criterion in the PARTNER trial [21]. The BiAV has elliptical annular morphology and tends to have an asymmetric calcium distribution, making valve deployment challenging for proper positioning and thus causing significant paravalvular regurgitation $[4,22]$. Nevertheless, many centers performed TAVR in patients with a BiAV and severe AS $[5,6,17,20]$. Hayashida et al. [5] showed that there were no significant differences in mortality and paravalvular leakage between patients with BiAV- and TAV-associated severe AS, whereas Costopoulos et al. [17] showed that there was a decreased survival rate in patients with a BiAV and severe AS after undergoing TAVR. Kochman et al. [18] and Bauer et al. [6] demonstrated that there were no survival differences, but there was an increased rate of paravalvular leakage in those with a BiAV compared with those with a TAV. A recently published study by Yousef et al. [20] found that TAVR had acceptable clinical outcomes in patients with severe AS and a BiAV, although the re-intervention rate was higher after TAVR due to valve embolization or migration. Recently, Yoon et al. [23] demonstrated that new-generation devices led to favorable clinical outcomes regarding mortality and paravalvular leakage in patients with a BiAV and severe AS. Nevertheless, all studies published until now regarding TAVR in patients with a BiAV focused mainly on valve replacement or dysfunction itself, without any concern for the accompanying pathology such as ascending aortopathy. This is the first report dealing with the changes in the ascending aorta's dimension after a successful TAVR procedure in patients with a BiAV and severe AS.

Two suggested hypotheses may explain why the as- cending aorta dilates in patients with a BiAV: i.e., inherent fragility and a hemodynamic burden. Patients with a BiAV were reported to have a larger aortic dimension than those with a TAV, and thus exposed to a higher risk of aortic dissection $[10,11]$, suggesting that BiAV-related aortopathy is an inherent disorder. This hypothesis was further advocated by the close association between the NOTCH1 gene and the development of BiAV [24]. Therefore, the current guideline recommend ascending aorta replacement should be considered even in asymptomatic BAV patients with maximal ascending aorta diameter greater than or equal to $50 \mathrm{~mm}$ with some risk factors [25]. However, a recent longitudinal study found that surgical AV replacement alone in patients with a BiAV and severe AS effectively prevents ascending aorta dilation [26], suggesting that a chronic hemodynamic burden may be an important contributor to the progression of ascending aortopathy that is found in patients with a BiAV. Although this finding should be confirmed in other cohorts, it is plausible that surgical AV replacement can prevent further dilation of the ascending aorta in patients with a BiAV and severe AS. However, this 'preventive' effect, if any, has never been suggested in the contemporary TAVR era.

We found that patients with a BiAV had a larger ascending aorta dimension pre-TAVR, compared with patients with a TAV and severe AS did, which is in agreement with earlier results [10,11]. This result confirms that the ascending aorta is inherently fragile in patients with a BiAV. However, the ascending aorta's dimension remained stable after TAVR in patients with a BiAV and severe AS, without a significant difference from that in TAV patients over 12-month of follow-up post-TAVR, highlighting that the same protective effect (already demonstrated through surgical AV replacement in a study by Kim et al. [26]) can be expected with TAVR in patients with a BiAV and severe AS. Therefore, based on the results of the current study, the progression of ascending aortopathy in patients with a BiAV and severe AS may be not a serious concern, although the follow-up period was relatively short.

This study has some limitations that should be acknowledged. First, the number of BiAV patients who were enrolled was relatively small, and the follow-up duration was relatively short. Therefore, this study should be considered a pilot study, and studies that recruit a 
larger number of BiAV patients with a longer follow-up period need to be performed to confirm the results. Second, BiAV patients with ascending aortic dilation larger than $50 \mathrm{~mm}$ were excluded because of ethical issues. Currently, surgical AV replacement is the first choice in patients with BiAV-associated severe AS, and ascending aorta surgery can be considered in patients with an ascending aorta's size larger than or equal to 50 $\mathrm{mm}$, mainly due to concern about the progression of ascending aortopathy and aortic dissection, depending on age, body size, etiology of valve disease and so on [25]. Finally, TTE was used to measure the diameter of the ascending aorta. TTE may be less accurate in patients with a poor echocardiographic window. However, in 67 patients recruited, the measurements were not significantly different from those using CT (Fig. 3). Nevertheless, CT may be more suited for longitudinal follow-up of the ascending aorta's size, and thus should be used in future studies to confirm the findings that were suggested here, although radiation issue is one of concern.

In conclusion, the growth rate of the ascending aorta's dimension after a TAVR procedure in patients with a BiAV and severe AS was at least similar to those with a TAV and severe AS. Therefore, concern over the progression of the ascending aorta's pathology after a successful TAVR procedure may not be needed, and TAVR could be considered in patients with a BiAV and severe AS with ascending aortic aneurysm. This study should lay a cornerstone for future large-scale studies with longer follow-up duration that recruit patients with a BiAV and severe AS complicated by ascending aortic aneurysm (i.e., ascending aorta size $>50 \mathrm{~mm}$ ).

\section{KEY MESSAGE}

1. Our single-center study demonstrated that concern over the progression of the ascending aorta's pathology after a successful transcatheter aortic valve replacement (TAVR) procedure may not be needed, and TAVR could be considered in patients with bicuspid aortic valve (BiAV) and severe aortic stenosis (AS) with the ascending aortic size less than $50 \mathrm{~mm}$.

2. Based on this study, future large-scale studies with longer follow-up duration that recruit pa- tients with a BiAV and severe AS complicated by ascending aortic aneurysm (i.e., ascending aorta size $>50 \mathrm{~mm}$ ) are warranted.

\section{Conflict of interest}

No potential conflict of interest relevant to this article was reported.

\section{REFERENCES}

1. Leon MB, Smith CR, Mack MJ, et al. Transcatheter or surgical aortic-valve replacement in intermediate-risk patients. N Engl J Med 2016;374:1609-1620.

2. Thourani VH, Kodali S, Makkar RR, et al. Transcatheter aortic valve replacement versus surgical valve replacement in intermediate-risk patients: a propensity score analysis. Lancet 2016;387:2218-2225.

3. Roberts WC, Janning KG, Ko JM, Filardo G, Matter GJ. Frequency of congenitally bicuspid aortic valves in patients $\geq 80$ years of age undergoing aortic valve replacement for aortic stenosis (with or without aortic regurgitation) and implications for transcatheter aortic valve implantation. Am J Cardiol 2012;109:1632-1636.

4. Himbert D, Pontnau F, Messika-Zeitoun D, et al. Feasibility and outcomes of transcatheter aortic valve implantation in high-risk patients with stenotic bicuspid aortic valves. Am J Cardiol 2012;110:877-883.

5. Hayashida K, Bouvier E, Lefevre T, et al. Transcatheter aortic valve implantation for patients with severe bicuspid aortic valve stenosis. Circ Cardiovasc Interv 2013;6:284-291.

6. Bauer T, Linke A, Sievert H, et al. Comparison of the effectiveness of transcatheter aortic valve implantation in patients with stenotic bicuspid versus tricuspid aortic valves (from the German TAVI Registry). Am J Cardiol 2014;113:518-521.

7. Fedak PW, Verma S, David TE, Leask RL, Weisel RD, Butany J. Clinical and pathophysiological implications of a bicuspid aortic valve. Circulation 2002;106:900-904.

8. Keane MG, Wiegers SE, Plappert T, Pochettino A, Bavaria JE, Sutton MG. Bicuspid aortic valves are associated with aortic dilatation out of proportion to coexistent valvular lesions. Circulation 2000;102(19 Suppl 3):III35-III39. 
9. Fernandes S, Khairy P, Graham DA, et al. Bicuspid aortic valve and associated aortic dilation in the young. Heart 2012;98:1014-1019.

10. Nistri S, Sorbo MD, Marin M, Palisi M, Scognamiglio $\mathrm{R}$, Thiene G. Aortic root dilatation in young men with normally functioning bicuspid aortic valves. Heart 1999;82:19-22.

11. Roberts CS, Roberts WC. Dissection of the aorta associated with congenital malformation of the aortic valve. J Am Coll Cardiol 1991;17:712-716.

12. Nishimura RA, Otto CM, Bonow RO, et al. 2014 AHA/ACC guideline for the management of patients with valvular heart disease: a report of the American College of Cardiology/American Heart Association Task Force on Practice Guidelines. J Am Coll Cardiol 2014;63:e57-e185.

13. Leon MB, Piazza N, Nikolsky E, et al. Standardized endpoint definitions for Transcatheter Aortic Valve Implantation clinical trials: a consensus report from the Valve Academic Research Consortium. J Am Coll Cardiol 2011;57:253-269.

14. Zoghbi WA, Chambers JB, Dumesnil JG, et al. Recommendations for evaluation of prosthetic valves with echocardiography and doppler ultrasound: a report from the American Society of Echocardiography's Guidelines and Standards Committee and the task force on prosthetic valves, developed in conjunction with the American College of Cardiology Cardiovascular Imaging Committee, Cardiac Imaging Committee of the American Heart Association, the European Association of Echocardiography, a registered branch of the European Society of Cardiology, the Japanese Society of Echocardiography and the Canadian Society of Echocardiography, endorsed by the American College of Cardiology Foundation, American Heart Association, European Association of Echocardiography, a registered branch of the European Society of Cardiology, the Japanese Society of Echocardiography, and Canadian Society of Echocardiography. J Am Soc Echocardiogr 2009;22:975-1014.

15. Hiratzka LF, Bakris GL, Beckman JA, et al. 2010 ACCF/ AHA/AATS/ACR/ASA/SCA/SCAI/SIR/STS/SVM guidelines for the diagnosis and management of patients with thoracic aortic disease. A report of the American College of Cardiology Foundation/American Heart Association Task Force on Practice Guidelines, American Association for Thoracic Surgery, American College of Radiology,American Stroke Association, Society of Cardiovascular Anes- thesiologists, Society for Cardiovascular Angiography and Interventions, Society of Interventional Radiology, Society of Thoracic Surgeons, and Society for Vascular Medicine. J Am Coll Cardiol 2010;55:e27-e129.

16. Elefteriades JA. Natural history of thoracic aortic aneurysms: indications for surgery, and surgical versus nonsurgical risks. Ann Thorac Surg 2002;74:S1877-S1880.

17. Costopoulos C, Latib A, Maisano F, et al. Comparison of results of transcatheter aortic valve implantation in patients with severely stenotic bicuspid versus tricuspid or nonbicuspid valves. Am J Cardiol 2014;113:1390-1393.

18. Kochman J, Huczek Z, Scislo P, et al. Comparison of oneand 12-month outcomes of transcatheter aortic valve replacement in patients with severely stenotic bicuspid versus tricuspid aortic valves (results from a multicenter registry). Am J Cardiol 2014;114:757-762.

19. Mylotte D, Lefevre T, Sondergaard L, et al. Transcatheter aortic valve replacement in bicuspid aortic valve disease. J Am Coll Cardiol 2014;64:2330-2339.

20. Yousef A, Simard T, Webb J, et al. Transcatheter aortic valve implantation in patients with bicuspid aortic valve: a patient level multi-center analysis. Int J Cardiol 2015;189:282-288.

21. Leon MB, Smith CR, Mack M, et al. Transcatheter aortic-valve implantation for aortic stenosis in patients who cannot undergo surgery. N Engl J Med 2010;363:1597-1607.

22. Chiam PT, Chao VT, Tan SY, et al. Percutaneous transcatheter heart valve implantation in a bicuspid aortic valve. JACC Cardiovasc Interv 2010;3:559-561.

23. Yoon SH, Lefevre T, Ahn JM, et al. Transcatheter aortic valve replacement with early- and new-generation devices in bicuspid aortic valve stenosis. J Am Coll Cardiol 2016;68:1195-1205.

24. Garg V, Muth AN, Ransom JF, et al. Mutations in NOTCH1 cause aortic valve disease. Nature 2005;437:270-274.

25. Erbel R, Aboyans V, Boileau C, et al. 2014 ESC guidelines on the diagnosis and treatment of aortic diseases: document covering acute and chronic aortic diseases of the thoracic and abdominal aorta of the adult. The Task Force for the Diagnosis and Treatment of Aortic Diseases of the European Society of Cardiology (ESC). Eur Heart J 2014;35:2873-2926.

26. Kim YG, Sun BJ, Park GM, et al. Aortopathy and bicuspid aortic valve: haemodynamic burden is main contributor to aortic dilatation. Heart 2012;98:1822-1827. 